\title{
Spindle cell carcinoma (sarcomatoid carcinoma) of maxillary sinus and nasal cavity with orbital involvement: A rare case report and brief review of literature
}

\author{
Serdar Altınay ${ }^{1 *}$, Ayse Altinok ${ }^{2}$, Pelin Altınok Süt ${ }^{3}$, Ümit Taskın $^{4}$ and Ahmet Bilici ${ }^{5}$ \\ ${ }^{1}$ Health Science University, Dr. Sadi Konuk Training and Research Hospital, Department of Pathology, Istanbul, Turkey \\ ${ }^{2}$ Medipol University, Department of Radiation Oncology, Istanbul, Turkey \\ ${ }^{3}$ Bezmi Alem University, Department of Radiation Oncology, Istanbul, Turkey \\ ${ }^{4}$ Health Science University, Bagcilar Training and Research Hospital, Department of ENT, Istanbul, Turkey \\ ${ }^{5}$ Medipol University, Department of Oncology, Istanbul, Turkey
}

\begin{abstract}
Sarcomatoid (spindle cell) carcinoma is a unique variant of squamous cell carcinoma. So far very rare cases of sarcomatoid carcinoma with maxillary origin have been reported in the Pubmed based research. A new case of spindle cell squamous cell carcinoma with ptosis is reporting in a 65-year old Turkish man. The mass lesion was mainly localized in the maxillary sinus, nasal cavity and extending towards the orbital wall. The cells had a large vesicular nucleus and marked macronucleoles. Tumor cells showed strong immunreactivity comprising both sarcomatous and epithelial components. Case was reported as sarcomatoid carcinoma of maxilla based on these characteristics. The patient was at stage $4 \mathrm{~b}$ in clinical staging and was regarded as non-operable. Intensity modulated radiotherapy technique was used and a regression at a rate of $1 / 3$ was observed in the tumor.
\end{abstract}

\section{Introduction}

Spindle cell carcinomas are a variant of squamous cell carcinoma, comprising both sarcomatous and epithelial components. These tumors, whose maxillary involvement was demonstrated by Leventon in 1981, have been documented in the upper aero-digestive tract, esophagus, salivary gland, thyroid, thymus, lungs, breasts, GIS, hepatobiliary system and the genitourinary tract. They occur quite rarely in the head/ neck region. They have been reported less frequently in the larynx, pharynx, oral cavity and the nasal region [1].

Maxillary involvement is limited to published cases (Table 1). As the number of cases is so low, there is no standard recommendation in the treatment protocol. These tumors with sarcomatoid components, which have a worse prognosis compared to squamous cell carcinoma of the maxilla, reach us at an advanced phase. To our knowledge, this is the first case of sarcomatoid carcinoma of the maxilla presenting as ptosis. Now a sarcomatoid carcinoma case with maxillary sinus and nasal cavity involvement and orbital extension is presenting and discussing the clinical-immunohistochemical findings and the positive response to radiotherapy along with literature data.

\section{Case history}

A 65-year old male patient applied to us complaining about the inability to breath from the left side of the nose since nearly 20 days, occasional bloody discharge and gradually growing swelling on the left side of the neck (Figure 1). There were no abnormalities in his anamnesis and family history. Irregular hemorrhagic masses were observed in the left nasal cavity at anterior rhinoscopy. A hard, fixed mass of approximately $3 \times 2 \mathrm{~cm}$ was detected in the left cheek. And a hard, fixed mass of approximately $5 \times 5 \mathrm{~cm}$, extending from the left submandibular region to the jugulodigastric region was palpated in the left submental and cervical area. In the MRI assessment performed in the face and neck region, soft tissue lesions at a diameter of $75 \times 55$ $\mathrm{mm}$ were observed, filling the left ethmoidal maxillary sinus in the left facial hemisphere and the left hemisphere region of the nasal cavity, appearing hypointense in T1 weighted images and hyperintense in T2 weighted images. Moreover, a soft tissue mass at a size of nearly $27 \times 15$ $\mathrm{mm}$, observed to be a continuation of this mass lesion, extending to the left orbital cavity, causing a marked push in the bulbus oculi and extraocular muscle plains, was noted. Lymph nodes, at a pathologic dimension were observed, with the larger ones having a size of 36x30 $\mathrm{mm}$, particularly noted in the left submandibular lodge in the left hemisphere of the neck (Figure 2).

Intranasal biopsy sample taken for diagnostic purposes comprised only the ulcer surface and the blood-fibrin mass. Thereupon, a second biopsy was taken. We observed a polypoid neoplasm, composed of atypical cells with ulcerated surface, large pleomorphic vesicular nuclei and marked macronucleoli, neighboring nasal mucosa

*Correspondence to: Serdar Altınay, MD, Associate Professor, Health Science University, Dr. Sadi Konuk Training and Research Hospital, Department of Pathology, Istanbul, Turkey, Tel: +90 (0) 212-414 6440; Fax: +90 (0) 21241442 52; E-mail: drserdara@yahoo.com

Key words: maxillary sinus, sarcomatoid carcinoma, spindle cell squamous cell carcinoma, radiotherapy

Received: September 06, 2018; Accepted: September 26, 2018; Published: September 28, 2018 
Altınay S (2018) Spindle cell carcinoma (sarcomatoid carcinoma) of maxillary sinus and nasal cavity with orbital involvement: A rare case report and brief review of literature

Table 1. Cases of sarcomatoid cancer of maxillary sinus reported in literature

\begin{tabular}{|c|c|c|c|}
\hline Authors & Age/sex & Stage & Treatment \\
\hline Leventon, et al. $1981[1]$ & - & 1 -superficial & - \\
\hline Asbury, et al.1992 [5] & $37 / \mathrm{F}$ & - & Surgery+post-operative RT \\
\hline Sadaba, et al. $2006[6]$ & $45 / \mathrm{M}$ & - & Chemoradioteraphy \\
\hline Howard, et al. $2007[7]$ & $54 / \mathrm{M}$ & T3N3M0 & Concurrent CT and RT \\
\hline Kumar, et al. $2008[4] *$ & $25 / \mathrm{M}$ & T4aN0M0 & RT \\
\hline Terada, et al. $2011[8]$ & $75 / \mathrm{M}$ & - & Surgery+Chemo radioteraphy \\
\hline Gupta, et al. $2013[(9]$ & $29 / \mathrm{M}$ & - & Only surgery \\
\hline Samuel, et al. $2013[10]$ & - & - & - \\
\hline Junaid, et al. (2017) [11] & $34 / \mathrm{F}$ & & Surgery+CT+RT \\
\hline $\begin{array}{c}\text { Altinay, } \text { et al. } 2017 \\
(\text { present case) }\end{array}$ & $65 / \mathrm{M}$ & T4bN1M0 & $\begin{array}{c}\text { Intensity modulated } \\
\text { radiotherapy+CT }\end{array}$ \\
\hline
\end{tabular}

CT: Chemoteraphy. RT: Radioteraphy. *Expanded version of table in Kumar, et al. [4]

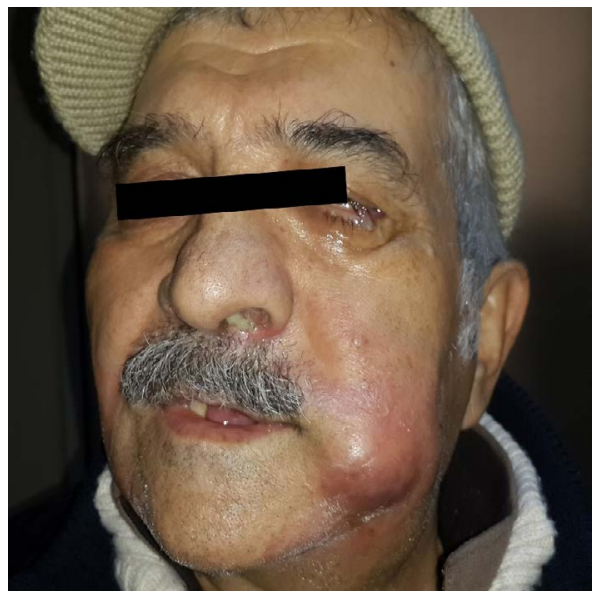

Figure 1. Swelling in the left cheek and redness

under microscopy. The tumor had no metaplastic mesenchymal components such as chondroid-osteoid. Although wide eosinophilic cytoplasms were observed sporadically, there were no keratin pearls for squamous cell carcinoma. Wide necrotic areas were noted in the stroma and mitotic count resulted to be $7-8 / 50 \mathrm{HPF}$. The tumor cells displayed a strong immunoreactivity with p63, CK5/6 and Vimentin and a strong immunoreactivity with Pan CK (AE1/AE3) in the immunohistochemical assessment (Figure 3). S100, HMB-45, MART1, SMA, Synaptophysin, LCA (CD45RO) and CD34 staining resulted to be negative. The diagnosis was made as sarcomatoid carcinoma due to the biphasic component verified also immunohistochemically. Similar atypical cells were detected in the fine needle aspiration performed on the lymph node in the left neck and were regarded as metastatic. Therefore, it was the first case of sarcomatoid carcinoma of the maxilla presenting as ptosis.

The treatment of the patient, who was at stage $4 \mathrm{~b}$ in clinical staging and was regarded as non-operable, was planned as follows. As the sarcomatous component was a subtype of the squamatous cell tumor, it was decided to perform a definitive radiotherapy along with chemotherapy to be applied once every three weeks for its radiosensitive effect. Cisplatin was not used due to the deficiency in renal functions. Instead, carboplatin was used once every 21 days at $100 \mathrm{mg} / \mathrm{m} 2 /$ day. The left paranasal sinus region and the bilateral neck of the patient were identified as the target volume for radiotherapy. Intensity modulated radiotherapy technique was used and it was planned to perform 6572 cGy radiotherapy in 31 fractions. The patient underwent treatment every day with daily imaging. A regression at a rate of $1 / 3$ was observed in the tumor in the weekly imaging (Figure 4).

\section{Discussion}

Sarcomatoid (spindle cell) carcinoma is a unique variant of squamous cell carcinoma [2]. The sarcomatoid component is formed due to the clonal proliferation of atypical spindle cells. Molecular studies have demonstrated that the tumors in the sarcomatoid carcinomas in the head/neck region develop with the transformation of the epithelial component [3].

Sarcomatoid carcinomas constitute approximately $1 \%$ of all squamatous cell carcinomas in the head/neck region [2]. They are most frequently observed in the larynx, while the regions where they are less localized are the hypopharynx, oropharynx, sinuses and the nasal cavity (1). As indicated in the table prepared by Kumar, et al. [4], the number of such cases is low [1,5-11]. Only seven cases with maxillary localization have been reported in literature [10].

There is male dominance/superiority in these tumors which develop mostly in the $5^{\text {th }}$ and $6^{\text {th }}$ decades. Our case, with maxillary sinus localization, is 65 years old and the second oldest patient among those reported in literature and is compliant with the male gender criterion. Thompson, et al. [2] reported a median age of 66 and a M/F ratio of 13:1 in the larynx related sarcomatoid carcinoma series of 187 subjects. In the study where they assessed 103 cases relating to the head/neck region with 6 being of maxillary origin, In the study where they assessed 103 cases relating to the head/neck region with 6 being of maxillary origin, Viswanathan, et al. [12] reported a median age of 53 and a M/F ratio of 3.7:1. Epithelial differentiation of tumor cells and staining for one or more epithelial markers supports the diagnosis. In most spindle carcinoma cases $(26 \%$ - 62\%) Keratin and cytokeratin are commonly found to be positive. Authors detected a Pan CK (intermediate-low MWCK) staining of $50 \%$ and a p63 staining of $30 \%$ in their study in which they provided details about the immunohistochemical
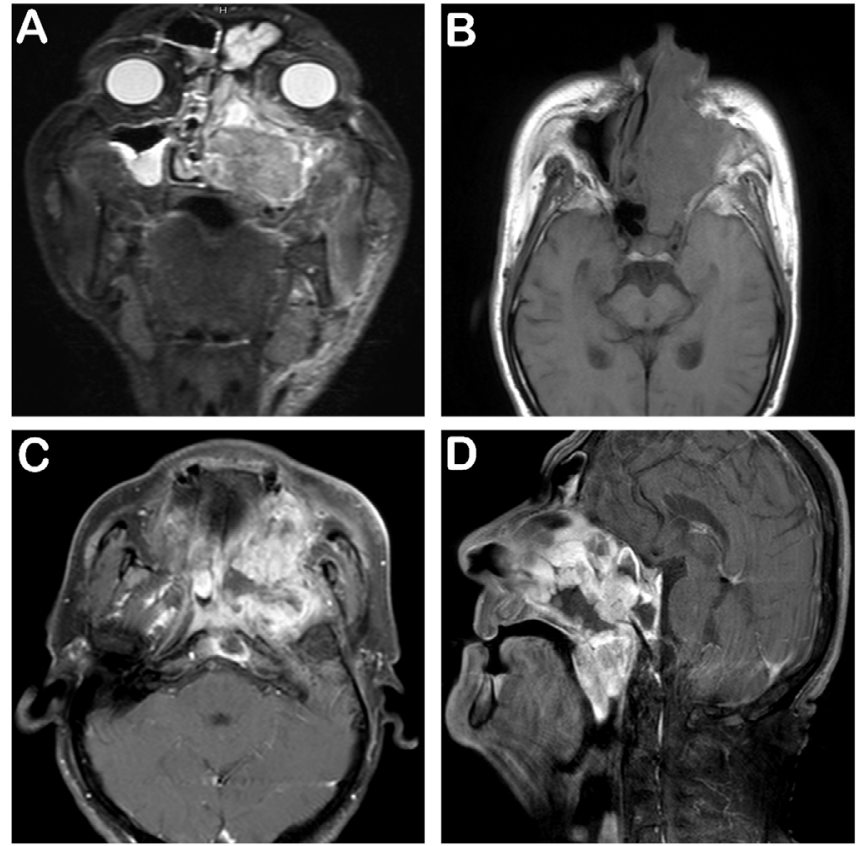

Figure 2. MRI images; 1A-T2 STIR coronal image: The lesion fills the left maxillary, frontal sinuses and left ethmoidal cellules. Left orbital intracanal space, left inferior oblique muscle and left hard palate are also invaded. 1B- T1 SE axial non-contrast image: Left nasal cavity, left half of the sphenoid sinus and left maxillary sinus are invaded. 1C- T1 SE axial contrast enhanced image: Clivus and left parapharyngeal invasion can be seen. 1D-T1 SE sagittal contrast enhanced image: Nasopharynx is obliterated with the bulk of mass lesion. Soft palate invasion is shown 

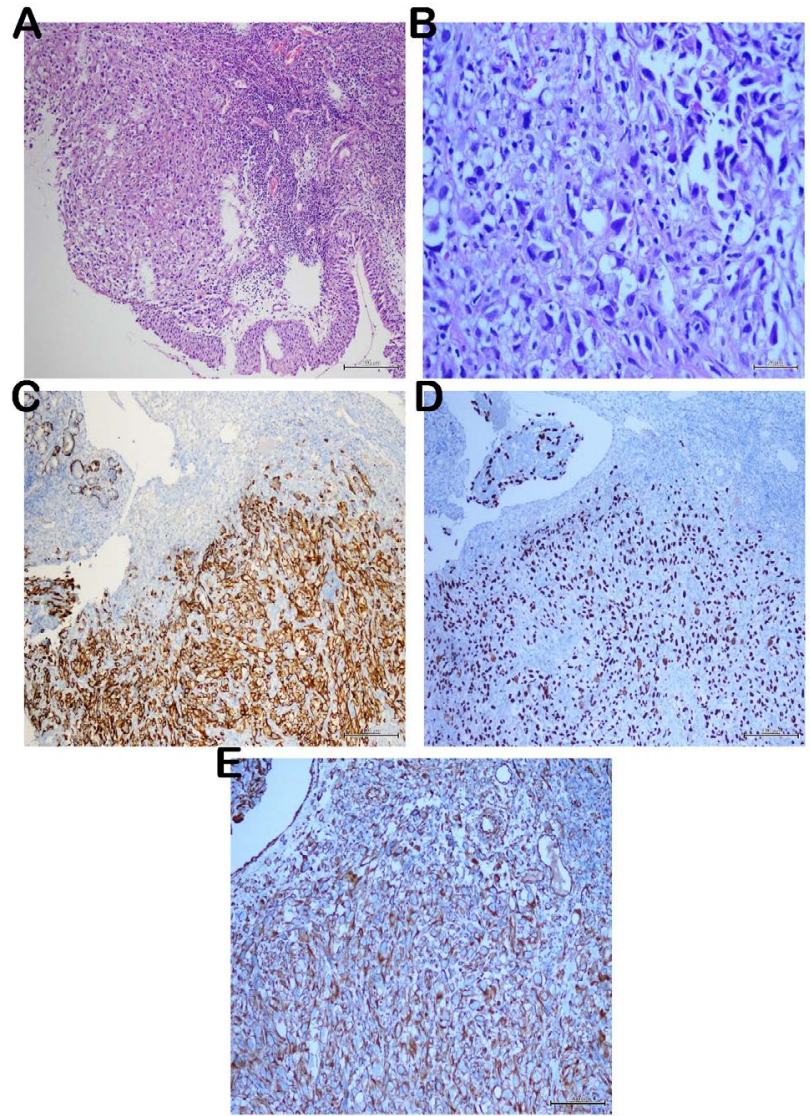

Figure 3. Histological features; atypical tumor cells are observed adjacent to the norma nasal mucosa $(\mathrm{A} ; \mathrm{H} \& \mathrm{E}, \mathrm{x} 100)$. Neoplastic tissue is composed of a prominent atypical morphology, with clear cytoplasms and hypercromatic pleomorphic nucleus in high powe (B;H\&E; 400$)$. Immünohistochemical features: Strong immünreactivity with CK5/6 and p63 (C-D;x100) and Vimentin in tumor cells (E;x200)
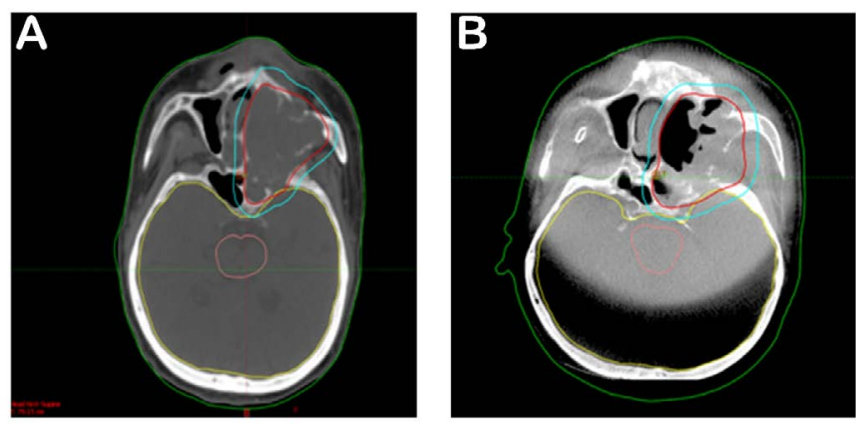

Figure 4. CT images: This image shows tumor volume before treatment. Red line is surrounding of tumor area (A). Second image displays treatment effect of radioteraphy (B). Please compare to Figure-4A, note decrease of tumor volume

characteristics. Furthermore, positivity to mesenchymal-type markers is also demonstrated. The authors reported the EMA staining as $17.9 \%$ (3) and $28.8 \%$ [12] and both authors published the Vimentin staining as $100 \%$. Tumor cells which have sarcomatous component are showed strong Vimentin immunreactivity in present case. Some spindle SCC lesions have also been shown to express markers of muscle differentiation including SMA and muscle specific actin [13]. But we didn't observed smoothle muscle differentation in tumor cells.

As the sarcomatoid component is associated with the worse prognosis, a major responsibility lays on the pathologist in the detection of this component. Vimentin staining holds a critical importance in the detection of mesenchymal component. The pathologist should also consider malignant mesenchymal tumors such as spindle cell melanoma, leiomyosarcoma and myoepithelial carcinoma included into the differential diagnosis of sarcomatoid carcinomas [12,13]. The implementation of a wide immunohistochemical panel will solve this problem. We have detected a strong positivity with CK5/6 among epithelial markers and a focally strong positivity with Pan CK. EMA resulted to be negative. HMB45, S100, MART-1 staining applied for melanoma displayed a negative immunoreactivity. Furthermore, SMA staining applied for leiomyosarcoma and CD34 staining applied for epithelioid angiosarcoma were negative.

Sarcomatoid carcinomas of the head/neck region display a polypoid configuration both in the case reports and in the series [2,12]. We have observed this polypoid development pattern in the macroscopic as well as microscopic assessments.

Use of tobacco use and alcohol drinking constitute an etiological cause in the sarcomatoid carcinomas just as in squamous cell cancers $[2,12]$. However, our patient is not a smoker. There was no exposure history to radiation which was blamed as another etiological agent in our patient. Viswanathan, et al. [12]. reported no radiation exposure in any of their 103-subject series.

Lymph node involvement of the neck may be found in maxillary sarcomatoid carcinomas generally presenting at an advanced stage [8]. It should also be noted that the cervical nodal metastasis incidence varies between $7.5 \%-26 \%$ in sarcomatoid carcinomas localized in the head and neck [12]. A high number of lymph nodes, with the largest one reaching a diameter of $3 \mathrm{~cm}$, was detected in the MRI assessment conducted on our patient. It was verified by needle biopsy that one of these lymph nodes was metastatic.

In the study comprising sarcomatoid carcinomas of the head and neck region, it was observed that 20 out of 39 patients followed up from 1 month up to 39 months ( 8 months on average) were disease-free, 6 had the disease, 8 experienced recurrence, 2 had metastasis in the lungs and soft tissue and 3 patients died (in the $1^{\text {st }}, 2^{\text {nd }}$ and $3^{\text {rd }}$ months after treatment) [12]

The follow-up periods are short in maxillary sarcomatoid carcinomas restricted to subjects. In published literature the treatment modality of spindle cell sarcoma follows same footsteps as that of squamous cell carcinoma of similar stage with wide surgical excision being the preferred choice of treatment. Nevertheless, when compared to squamous cell carcinoma, within the head and neck region, spindle cell carcinoma is considered as a more aggressive tumor and has a propensity to recur and metastasize early. Thus, there is still no standard achieved in the therapeutic decision. CT and RT was performed in consequence to right maxillary sinus resection in the patient with the longest known follow-up period and he was reported to be alive 5 years following the initial presentation [9]. Although there is no definitive therapeutic method, the primary recommendation of guidelines is surgical removal in the treatment of maxillary sinus localized tumors. Consequently, adjuvant radiotherapy and/or chemotherapy is performed upon evaluating the characteristics of the tumor and the adequacy of surgical exploration. Due to this ambiguity, we have decided on the treatment of our subject upon taking into account the localization of the tumor and the pathological subtype. Surgical resection and postoperative chemoradiotherapy constitute the standard treatment in all $\mathrm{T}$ stages (except for $\mathrm{T} 4 \mathrm{~b}$ ) of maxillary sinus localized tumors [14]. Our case was evaluated as T4b at diagnosis 

literature

due to nasopharyngeal, orbita and clivus involvement. Pathological lymphadenopathies were detected radiologically in the bilateral neck. There are proposals compiled from the outcomes of retrospective studies for classical squamous cell carcinomas at stage T4bN2M0 literature. These recommendations propose the use of chemotherapy agents with a radiosensitizing impact along with high dose radiation therapy. Until now, molecular imaging has had a limited value for the selection of nodal (gross tumor volume) GTV, but there are increasing evidences that both FDG positron emission tomography and diffusionweighted magnetic resonance imaging has a potential value for the delineation of the primary tumor GTV, effecting on dose distribution. The planning of the radiotherapy to be applied with the intensity modulated radiotherapy technique reduces the side effects that may arise in the patient and provides the opportunity to raise the dose to be administered [14]. We have applied radiotherapy with this technique in 31 fractions on our patient in a manner so as to make the biological equivalent dose $78 \mathrm{~Gy}$ in 31 fractions. Our patient is still alive in the $44^{\text {th }}$ month following treatment. After treatment noted decrease of tumor volume. Authors point out that validation trials are ongoing, and in the coming years, one may expect to position the dose painting approach in the armamentarium for the treatment of patients with head-and-neck squamous cell carcinoma [14]

In conclusion, sarcomatoid carcinoma of maxilla is a rare entity in head and neck and it is a potentially aggressive tumor with high recurrence rate. We have presented our 65 -year old male patient having a significantly rare sarcomatoid carcinoma localized maxillary sinus and nasal cavity. To our knowledge, this is the first case of sarcomatoid carcinoma of the maxilla presenting as ptosis also have a positive response to radiotherapy.

Written constent form is obtained from patient.

\section{Take home messages}

- The possibility of a maxillary sinus localized tumor should be considered in elder male patients applying with a swollen face and nasal congestion,

- Although the overlapping of histopathological characteristics of spindle cell tumors especially in the head/neck area poses a challenge in diagnosis, the recognition and verification of the sarcomatoid component lays a major responsibility on pathologists.

- Due to the fact that it has both epithelial and mesenchymal tissue, it can be a diagnostic problem for the pathologist. Immunohistochemical stains have an important role to play in its differentiation with other tumors.
- Sharing of cases where morphological-molecular characteristics are identified, which comprise a treatment modality and are followed up since a long time, will provide benefit in guiding the treatment of these tumors correctly.

\section{References}

1. Leventon GS, Evans HL (1981) Sarcomatoid squamous cell carcinoma of the mucous membranes of the head and neck: a clinicopathologic study of 20 cases. Cancer 48: 994-1003. [Crossref]

2. Thompson LD, Wieneke JA, Miettinen M, Heffner DK (2002) Spindle cell (sarcomatoid) carcinomas of the larynx: a clinicopathologic study of 187 cases. $\mathrm{Am} \mathrm{J}$ Surg Pathol 26: 153-170. [Crossref]

3. Stucky A, Sedghizadeh PP, Mahabady S, Chen X, Zhang C, et al. (2017) Single-cell genomic analysis of head and neck squamous cell carcinoma. Oncotarget 8: 7320873218. [Crossref]

4. Kumar M, Goyal S, Bahl A, Das P, Sharma DN, et al. (2008) Sarcomatoid carcinoma of the maxillary sinus: a rare head and neck tumor. $J$ Cancer Res Ther 4: 131-133. [Crossref]

5. Asbury L, Candelaria S, Rudak F, Stutzman CD, Lake DE (1992) High dose rate treatment of a maxillary sarcomatoid carcinoma: a case report. Med Dosim 17: 129133. [Crossref]

6. Sadaba LM, García-Layana A, Garcia-Gomez PJ, Salinas-Alaman A (2006) Sarcomatoid carcinoma and orbital apex syndrome. Eur J Ophthalmol 16: 608-610. [Crossref]

7. Howard SN, Bond WR, Hong IS, Foss RD (2007) Right maxillary sinus sarcomatoid carcinoma (sarcomatoid/spindle cell carcinoma). Otolaryngol Head Neck Surg 137: 355-357. [Crossref]

8. Terada T, Kawasaki T (2011) Spindle cell carcinoma of the nasal cavity. Int $J$ Clin Oncol 16: 165-168. [Crossref]

9. Gupta S, Santoriello D, Wieczorek R, De Lacure MD (2013) Spindle cell carcinoma of the nasal cavity. Rare Tumors 5: 10. [Crossref]

10. Samuel S, Sreelatha SV, Hegde N, Nair PP (2013) Spindle cell carcinoma in maxilla. BMJ Case Rep 2013. [Crossref]

11. Junaid M, Kazi M, Qadeer S (2017) Spindle cell carcinoma of the maxilla: A case report of rare entity. in Surgical Science 8: 220-227.

12. Viswanathan S, Rahman K, Pallavi S, Sachin J, Patil A, Chaturvedi P, et al. (2010) Sarcomatoid (spindle cell) carcinoma of the head and neck mucosal region: a clinicopathologic review of 103 cases from a tertiary referral cancer centre. Head Neck Pathol 4: 265-275. [Crossref]

13. Romañach MJ, Azevedo RS, Carlos R, de Almeida OP, Pires FR (2010) Clinicopathological and immunohistochemical features of oral spindle cell carcinoma. J Oral Pathol Med 39: 335-341. [Crossref]

14. Grégoire V, Thorwarth D, Lee JA (2018) Molecular imaging-guided radiotherapy for the treatment of head-and-neck squamous cell carcinoma: Does it fulfill the promises? Semin Radiat Oncol 28: 35-45. [Crossref]

Copyright: (C2018 Altınay S. This is an open-access article distributed under the terms of the Creative Commons Attribution License, which permits unrestricted use, distribution, and reproduction in any medium, provided the original author and source are credited. 